tiation, as this subject is known, have been notably rewarded where microbial systems have been examined; in particular, the divers processes of sporulation found in micro-organisms have interested many workers. A convenient approach to the study of sporulation involves the use of metabolic inhibitors which interfere with protein or nucleic acid syntheses. Experiments of this type can provide precise information on the process of induction and define the temporal parameters of the morphogenetic sequence. The investigations of Young and Fitz-James and Aronson exemplify this stratagem and have indicated the major changes in protein and nucleic acid metabolism which occur during endosporulation in Bacillus.

In the imperfect fungus Trichoderma conidia are light-induced, a fact familiar to mycologists for many years. This fungus has been taken by Galun and Gressel as a model system for analysing the involvement of nucleic acids in morphogenesis. Galun and Gressel observed a minimum growth period of $16 \mathrm{~h}$ before this mould was capable of responding to light induction and that conidiation was prevented by fluorouracil (FU) at concentrations which did not inhibit growth (Science, 151, 696; 1966). Subsequently, these workers found that an hour's treatment with FU would completely suppress sporulation up to $7 \mathrm{~h}$ after light induction (Developmental Biol., 15, 575; 1967). Once conidiophore branching was completed, however, after about $11 \mathrm{~h}$, sporulation always proceeded although at a reduced level. This aspect of Trichoderma differentiation is very reminiscent of the "point of no return" concept in the water mould Blastocladiella and the phase of "spore commitment" in Bacillus species.

Suppression of sporulation was reversed by uracil, though the uracil appeared to exert an additive rather than the expected competitive effect on. fluorouracil action. Uridine also affected reversal of FU inhibition, but, while the tritium label from uridine appeared both in uridylic and cytidylic acid peaks, the carbon-14 label from the analogue was found only in the uridylicfluorouridylic acid peaks. Fluorouracil suppresses sporulation by its specific action on RNA and it is noteworthy that indefinite growth can continue with a selective inhibition of this morphogenetic pathway. All species of RNA were shown. to contain FU and additionally a new $s$ RNA was eluted from methylated albumin columns between the $4 S$ and $5 S$ species. Pulse labelling with FU or uracil yielded a similar RNA elution profile suggesting that the analogue was incorporated into $m \mathrm{RNA}$. Of a large number of other RNA antimetabolites tested, only 8-azaguanine produced results similar to fluorouracil and induced synthesis of the exotic sRNA species.

At the present time the only substantial conclusion to be drawn from these experiments is that the photoinduced sporulation in Trichoderma is RNA mediated. The suppression of this specific morphogenetic sequence could be via FU-sRNA, FU-mRNA, via both, or via yet another mechanism. Extended studies of these fraudulent nucleic acids will doubtless lead to important findings on the molecular control of growth and morphogenesis, and future reports from this group will be awaited with considerable anticipation. In particular, the fact that pre-induced cultures can be partially suppressed by FU encourages the hope that significant data may be forthcoming on the nature of the light induction mechanism.

\section{Mouse Satellite DNA}

\section{from a Correspondent in Cell Biology}

DENSITY gradient centrifugation separates mouse nuclear DNA into two components: a major component, about 90 per cent of the total, and a minor component, satellite DNA, with a slightly lower buoyant density. This satellite DNA has some very remarkable properties. It is unusually homogeneous in $\mathrm{G}+\mathrm{C}$ content (Flamm et al., 1966) and undergoes very rapid renaturation at low DNA concentrations (Walker and MacLaren, 1965). Waring and Britten (Science, 154, $799 ; 1966)$ found heat denatured satellite DNA renatures faster than any of the other animal, bacterial and viral DNAs that they examined. It even renatures fifteen times faster than simian virus 40 DNA which only has 6,000 base pairs, so satellite DNA must form a homogeneous population of molecules with a repeated sequence of only about $300-400$ base pairs. Waring and Britten suggested each mouse genome probably contains about one million copies of this short sequence.

Flamm, McCallum and Walker at Edinburgh have recently achieved the separation of the two complementary strands of the satellite DNA duplex and report on their properties (Proc. Nat. Acad. Sci., 5\%, $1729 ; 1967)$. After isolating the satellite DNA from the major component by gradient centrifugation they re-ran the satellite band in a caesium chloride gradient at $p \mathrm{H} 12 \cdot 5$. At this high $p \mathrm{H}$ the DNA duplex is separated into its two strands and these have different buoyant densities. The heavy strand is rich in purimidines $C+T$, and the light strand in the purines $A+G$. The two strands have complementary base compositions and the renaturation characteristics of equimolar mixtures are very similar to those of native satellite DNA after heat denaturation. Neither heavy nor light strands alone will renature.

Interestingly, although some sequence homologies exist between the total DNAs of mice, rats and guinea-pigs, mouse satellite DNA does not renature with total guinea-pig or rat DNA which includes the satellite DNA of these species. It appears that the nucleotide sequences of satellite DNA are completely species specific.

This work is the first in which the complementary strands of DNA from a higher organism have been isolated and the very fact that this can be done implies that satellite DNA is homogeneous and has only a short base sequence. This does not mean, however, that satellite DNA is of low molecular weight. Flamm and his collaborators have isolated molecules with a molecular weight of 40 million, indicating at least 200 repeating sequences of $300-400$ base pairs strung end to end. This makes it highly unlikely that satellite DNA is viral or proviral in origin, for it seems incon.ceivable that a virus could exist with such a genome. Other properties of mouse satellite DNA, its presence in all the strains of mice and tissues investigated, its association with metaphase chromosomes (Maio and Schildkraut, J. Mol. Biol., 24, 29; 1967) and its metabolic stability, also argue against a viral origin.

What is the function of satellite DNA ? It is unlikely to code for protein and yet it forms 10 per cent of the cell's total DNA. What possible purpose is served by having so many, apparently identical, short sequences within the same genome? 